\title{
Aplicação de fórmula corretiva nas alterações da pressão intraocular dos pacientes submetidos a LASIK
}

\author{
Application of corrective formula for intraocular pressure changes in \\ patients that underwent LASIK
}

Thiago George Cabral Silva ${ }^{1}$, Júlia Gomes Fernandes Polido², Maurício Vieira Pinheiro ${ }^{3}$, André Luís de Freitas Silva ${ }^{3}$, Laerte Goldbach ${ }^{3}$, Vera lúcia Degaspare Monte Mascaro ${ }^{4}$, Pedro Durães Serracarbassa ${ }^{5}$, Maria Emília Xavier dos Santos Araújo 4

\section{RESUMO}

Objetivo: Comparar a pressão intraocular (PIO) pré e pós-LASIK, correlacionando-as com as mudanças da espessura corneana central (ECC) e ceratometria simulada média (K), assim como verificar o resultado de fórmula corretiva proposta anteriormente.

Métodos: Estudo longitudinal, prospectivo, realizado em pacientes submetidos a LASIK. Os pacientes foram submetidos ao exame oftalmológico completo, no préoperatório e após 2 meses da cirurgia. A pressão intraocular foi avaliada com tonômetro de aplanação de Goldmann entre $9 \mathrm{~h}$ e $11 \mathrm{~h}$ da manhã, a ceratometria simulada média foi avaliada por meio de topografia corneana e a espessura corneana central foi aferida por paquímetro ultrassônico, sendo considerada a média de três aferições. Foram excluídos dois pacientes com cirurgias ou doenças oculares prévias, e uso prévio de corticosteróide tópico nos últimos três meses. As cirurgias foram realizadas de acordo com os procedimentos-padrão. Foi utilizada a fórmula [PIO real = $\mathrm{PIO}$ aferida $+(540-\mathrm{ECC}) / 71+(43-\mathrm{K}) / 2,7+0,75 \mathrm{mmHg}]$ proposta para correção da pressão intraocular pós-operatória.

Resultados: Quinze olhos de oito pacientes foram avaliados, a idade variou de 24 a 46 anos (média: $31,37 \pm 7,27)$. Foi observada diferença estatisticamente significante entre as medidas da pressão intraocular, de ceratometria simulada média e da espessura corneana central pré e pós-LASIK. ( $p=0,0001)$. Foi observado que para cada 1D corrigida, há uma subestimação, em média, de 1,06 $\pm 0,59 \mathrm{mmHg}(0,11$ a 1,89 mmHg). A aplicação da fórmula corretiva levou a $80 \%$ dos olhos com a tonometria estimada entre $\pm 2,50 \mathrm{mmHg}$ da pré-operatória, no entanto, quando comparada com a tonometria pré-operatória, estas são estatisticamente diferentes $(p=0,0266)$.

Conclusões: Os olhos submetidos a LASIK apresentaram PIO pós-operatória menor do que a pré-operatória. A pressão intraocular pôde ser moderadamente correlacionada com a espessura corneana central e fracamente com a ceratometria simulada média. Não houve correlação entre a profundidade de ablação e a variação da pressão intraocular, no pós-operatório. Usando a fórmula proposta, pudemos averiguar que $80 \%$ dos pacientes apresentaram pressão intraocular entre $\pm 2,50 \mathrm{mmHg}$ da pré-operatória.

Descritores: Pressão intraocular/fisiologia; Ceratomileuse assistida por excimer laser in situ; Lasers de excimer; Tonometria ocular; Topografia da córnea; Procedimentos cirúrgicos refrativos

\begin{abstract}
Purpose: To compare the intraocular pressure (IOP) pre and post LASIK, correlating it to changes in central corneal thickness (CCT) and average simulated keratometry (K), as well as verifying the results of a corrective formula previously proposed.

Methods: Longitudinal prospective study conducted in outpatients that underwent to LASIK. Patients underwent complete ophthalmic examination, previously and 2 months after the surgery. Intraocular pressure was evaluated with Goldmann applanation tonometer between $9 \mathrm{am}$ and $11 \mathrm{am}$, average simulated keratometry was evaluated using corneal topography and central corneal thickness was measured with ultrasound pachymetry, been considered the average of three measurements. Two patients were excluded due to surgery or eye disease, and previous use of topical steroids over the past three months. The surgeries were performed according to standard procedures. The formula [real IOP $=1 O P$ measured $+(540-E C C) / 71+(43-K) / 2.7+0.75 \mathrm{mmHg}]$ proposed for correcting intraocular pressure was used.

Results: Fifteen eyes of eight patients were evaluated, age ranged from 24 to 46 years (mean: $31.37 \pm 7.27$ ). There was a statistically significant difference between the measurements of intraocular pressure, central corneal thickness and average simulated keratometry pre and post-LASIK. $(p=0.0001)$. It was observed that each 1D corrected underestimated the IOP $1.06 \pm 0.59 \mathrm{mmHg}(0.11 \mathrm{a} 1.89 \mathrm{mmHg})$. The use of the corrective formula lead to $80 \%$ of eyes within $2.50 \mathrm{mmHg}$ of preoperative intraocular pressure. Although, the two sets of data are statistically different $(p=0.0266)$.

Conclusions: POSt LASIK eyes presented lower intraocular pressure than preoperatively. Intraocular pressure was moderately correlated to central corneal thickness and weakly correlated to average simulated keratometry. With the use of the corrective formula, we were able to determine that $80 \%$ were within $2.50 \mathrm{mmHg}$ of the preoperative intraocular pressure.
\end{abstract}

Keywords: Intraocular pressure/physiology; Keratomileusis, laser in situ; Lasers, excimer; Photorefractive keratectomy; Tonometry, ocular; Corneal topography; Refractive surgical procedures

\section{INTRODUÇÃO}

Desde 1993, LASIK (laser in situ keratomileusis) é a técnica cirúrgica mais executada pelos cirurgiões refrativos ${ }^{(1)}$, não só pela rápida recuperação visual, como também pela possibilidade de correção das ametropias mais altas ${ }^{(2)}$. A correção é obtida pela alteração da curvatura anterior da córnea, havendo diminuição da sua espessura secundária à ação do laser. Entretanto, essas mudanças estruturais estão correlacionadas com leituras subestimadas da pressão intraocular (PIO) no pós-operatório pelo tonômetro de aplanação de Goldmann (TAG) ${ }^{(3)}$.
Submitted for publication: August 12,2010

Accepted for publication: March 14, 2011

Study carried out at the Departamento de Oftalmologia - Hospital do Servidor Público Estadual de São Paulo - HSPE-SP/IAMSPE.

Postgraduate student, Hospital do Servidor Público Estadual de São Paulo - HSPE-São Paulo(SP)- Brasil. Medical Resident, Hospital do Servidor Público Estadual de São Paulo - HSPE - São Paulo (SP) - Brasil. 3 Physician, Hospital do Servidor Público Estadual de São Paulo - HSPE - São Paulo (SP) - Brasil.

${ }^{4}$ Physician, Universidade Federal de São Paulo - UNIFESP - São Paulo (SP) - Brasil.

Physician, Universidade de São Paulo - USP - São Paulo (SP) - Brasil.
Funding: No specific financial support was available for this study.

Disclosure of potential conflicts of interest: T.G.C.Silva, None; J.G.F.Polido, None; M.V.Pinheiro, None; A.L.F.Silva, None; L.Goldbach, None; V.L.D.M.Mascaro, None; P.D.Serracarbassa, None; M.E.X.S.Araújo, None.

Correspondence address: Thiago George Cabral Silva. Departamento de Oftalmologia HSPE/SP Av. Ibirapuera, 981 - São Paulo - SP - 04029-000 - Brazil

E-mail: thiagogeorge@hotmail.com 
Há uma correlação entre miopia e glaucoma, e nos altos míopes, alterações do nervo óptico e campo visual são mais difíceis de serem avaliadas, tendo então a PIO um papel importantíssimo na avaliação e acompanhamento do glaucoma em pacientes com esse tipo de ametropia ${ }^{(4)}$.

A TAG ainda é considerada o padrão-ouro na medida da PIO. Autores já demonstraram a variação da estimação da PIO com a variação da espessura central corneana (ECC) aferidas pelo TAG (5).

Recentemente, uma nova variável foi apontada como mais um fator de influência na variação da medida da PIO em pacientes pósLASIK, a instabilidade do flap ${ }^{(3)}$. Levando em consideração a ECC, ceratometria media $(K)$ e um fator de correção para a instabilidade do flap corneano, em 2006 foi proposta uma fórmula corretiva para a estimativa da $\mathrm{PIO}$ em pacientes submetidos a $\mathrm{LASIK}^{(3)}$.

O objetivo desse trabalho é comparar a PIO pré e pós-LASIK, correlacionando-as com as mudanças da ECC e K, assim como verificar se a fórmula proposta anteriormente ${ }^{(3)}$ é aplicável aos nossos pacientes.

\section{MÉTODOS}

Estudo longitudinal, prospectivo, realizado em pacientes do ambulatório de Doenças Externas e Córnea do Hospital do Servidor Público Estadual de São Paulo (HSPE-SP) submetidos ao LASIK com o Excimer Nidek ${ }^{\circledR}$. Aprovado pelo comitê de ética em pesquisa do Hospital do Servidor Público Estadual de São Paulo (HSPE/IAMSPE-SP). Todos os pacientes assinaram um termo de consentimento informado individual.

Foram incluídos 15 olhos de 8 pacientes com miopia ou astigmatismo miópico, com equivalente esférico (EE) menor que -10,00 D, K pós-operatória mínima estimada de 36,00 D além de ECC maior ou igual a $500 \mu \mathrm{m}$, no pré-operatório e pós-operatória mínima estimada de $350 \mu \mathrm{m}$. Foram excluídos dois pacientes com cirurgias ou doenças oculares prévias, e uso prévio de corticosteróide tópico nos últimos três meses. Doenças sistêmicas e gravidez não foram admitidas.

Os pacientes foram submetidos, no pré-operatório, ao exame oftalmológico que incluiu: acuidade visual medida com a tabela de Snellen, refratometria dinâmica e cicloplegiada, PIO, obtida por meio de TAG, entre $9 \mathrm{~h}$ e $11 \mathrm{~h}$ da manhã, biomicroscopia anterior e posterior (mácula, nervo óptico) e oftalmoscopia binocular indireta (retina periférica). O exame oftalmológico foi normal em todos os casos.
A topografia corneana e a ceratometria simulada foram obtidas pelo aparelho EyeSys ${ }^{\circledR}$ 2000. A ECC foi aferida com paquímetro ultrassônico Allergan-Humphrey ${ }^{\circledR}$ modelo 850, sendo considerada a média de três aferições consecutivas.

As cirurgias foram realizadas de acordo com os procedimentos padrão, em resumo: marcação corneana, colocação do anel de sucção, confirmação da PIO com tonômetro de Barraquer, confecção do flap corneano de 160 um de espessura e 9,5 mm de diâmetro com microcerátomo Nidek MK $2000^{\circledR}$ e rebatido do lado nasal, fotoablação com excimer laser Nidek EC $500{ }^{\circledR}$ com zona de ablação de $6,0 \mathrm{~mm}$. Colírio de dexametasona 0,1\% + moxifloxacino 0,5\% (Vigadexa ${ }^{\circledR}$, Alcon, Brasil) foi usado 4 vezes ao dia, nos 10 primeiros dias, concomitante ao uso de lubrificante tópico 5x/dia por 90 dias (Fresh Tears ${ }^{\circledR}$, Allergan, Brasil). A PIO foi aferida após dois meses da cirurgia entre 9 e 11 h da manhã.

A fórmula utilizada foi: $\mathrm{PIO}$ real $=\mathrm{PIO}$ aferida $+(540-\mathrm{ECC}) / 71+$ $(43-\mathrm{K}) / 2,7+0,75 \mathrm{mmHg}^{(3)}$.

Teste $t$ pareado (Student), análise de regressão e correlação de Pearson foram analisados pelo programa estatístico SPSS (14.0, Chicago IL). Valor de $\mathrm{p} \leq 0,05$ foi considerado estatisticamente significante. Os resultados são apresentados como Média \pm Desvio Padrão (Valor Mínimo a Valor Máximo).

\section{RESULTADOS}

Quinze olhos de oito pacientes foram submetidos a LASIK. A idade média foi 31,3 \pm 7,3 anos (24 a 46 anos). Apresentavam miopia ou astigmatismo miópico, com equivalente esférico de -5,18 $\pm 2,47 \mathrm{D}(-2,5$ a $-9,5 \mathrm{D})$. Cinco pacientes eram do sexo feminino (62,5\%). A acuidade visual pós-operatória foi $\geq 20 / 20$ AO em todos os pacientes. A K no pré-operatório foi de 42,80 $\pm 1,09 \mathrm{D}(41,61$ a 45,12 D) e no pós-operatório foi de 39,20 \pm 1,78 D (36,80 a 42,93 D). A variação foi de $-3,60 \pm 1,41 \mathrm{D}(1,63$ a 6,52 D). A média de ECC no pré-operatório foi de 549,80 $\pm 25,28 \mu \mathrm{m}(507$ a $596 \mu \mathrm{m})$ e no pósoperatório de 480,13 $\pm 33,66 \mu \mathrm{m}$ (430 a $490 \mu \mathrm{m})$. A redução da ECC foi de $69,67 \pm 31,31 \mu \mathrm{m}$ (20 a $129 \mu \mathrm{m})$. A PIO foi 17,13 $\pm 2,41 \mathrm{mmHg}$ (14 a $22 \mathrm{mmHg}$ ) e $12,67 \pm 2,50 \mathrm{mmHg}$ (9 a $18 \mathrm{mmHg}$ ), no pré e pós-operatório, respectivamente. A variação da PIO foi de $-4,47 \pm$ $2,07 \mathrm{mmHg}$ (Tabela 1). Foi observada diferença estatisticamente

Tabela 1. Base de dados

\begin{tabular}{|c|c|c|c|c|c|c|c|c|c|c|c|c|c|}
\hline Olho & Sexo & Idade & $\begin{array}{l}\text { ECC Pré } \\
(\mu \mathrm{m})\end{array}$ & $\begin{array}{c}\text { ECC Pós } \\
(\mu \mathrm{m})\end{array}$ & $\begin{array}{c}\Delta \text { ECC } \\
(\mu \mathrm{m})\end{array}$ & $\begin{array}{c}\text { Ablação } \\
(\mu \mathrm{m})\end{array}$ & $\begin{array}{l}\text { PIO Pré } \\
(\mathrm{mmHg})\end{array}$ & $\begin{array}{l}\text { PioPós } \\
(\mathrm{mmHg})\end{array}$ & $\begin{array}{c}\Delta \mathrm{PIO} \\
(\mathrm{mmHg})\end{array}$ & $\begin{array}{l}\text { KPré } \\
\text { (D) }\end{array}$ & $\begin{array}{l}\text { KPós } \\
\text { (D) }\end{array}$ & $\begin{array}{r}\Delta \mathrm{K} \\
\text { (D) }\end{array}$ & $\begin{array}{l}\mathrm{EE} \\
\text { (D) }\end{array}$ \\
\hline OD & $\mathrm{F}$ & 28 & 534 & 450 & 84 & 64,8 & 15 & 10 & 5 & 41,93 & 38,48 & 3,45 & $-3,50$ \\
\hline OE & $\mathrm{F}$ & 28 & 525 & 460 & 65 & 61,5 & 16 & 10 & 6 & 41,83 & 38,35 & 3,48 & $-3,50$ \\
\hline OD & $\mathrm{F}$ & 27 & 507 & 450 & 57 & 87,0 & 14 & 9 & 5 & 45,12 & 41,25 & 3,87 & $-6,00$ \\
\hline $\mathrm{OD}$ & $M$ & 36 & 560 & 505 & 55 & 81,3 & 21 & 14 & 7 & 42,45 & 39,33 & 3,12 & $-5,00$ \\
\hline OE & $M$ & 36 & 560 & 500 & 60 & 77,6 & 22 & 13 & 9 & 42,90 & 39,75 & 3,15 & $-4,75$ \\
\hline OD & $\mathrm{F}$ & 33 & 550 & 490 & 60 & 73,4 & 17 & 12 & 5 & 42,08 & 38,88 & 3,20 & $-4,50$ \\
\hline OE & $\mathrm{F}$ & 33 & 543 & 490 & 53 & 73,5 & 14 & 10 & 4 & 42,45 & 38,48 & 3,97 & $-4,50$ \\
\hline OD & $\mathrm{F}$ & 24 & 525 & 505 & 20 & 41,1 & 17 & 13 & 4 & 41,61 & 39,98 & 1,63 & $-2,25$ \\
\hline OE & $\mathrm{F}$ & 24 & 525 & 504 & 21 & 41,3 & 17 & 13 & 4 & 42,34 & 40,37 & 1,97 & $-2,25$ \\
\hline OD & $M$ & 46 & 560 & 450 & 110 & 124,0 & 19 & 17 & 2 & 42,02 & 37,70 & 4,32 & $-8,00$ \\
\hline OE & $M$ & 46 & 569 & 440 & 129 & 124,0 & 18 & 12 & 6 & 42,02 & 37,04 & 4,98 & $-8,00$ \\
\hline OD & $F$ & 24 & 596 & 534 & 62 & 60,0 & 15 & 12 & 3 & 44,00 & 41,46 & 2,54 & $-3,00$ \\
\hline OE & $\mathrm{F}$ & 24 & 596 & 534 & 62 & 62,0 & 15 & 13 & 2 & 44,80 & 42,93 & 1,87 & $-3,50$ \\
\hline OD & $M$ & 30 & 552 & 460 & 92 & 95,1 & 19 & 18 & 1 & 43,32 & 36,80 & 6,52 & $-9,50$ \\
\hline $\mathrm{OE}$ & $M$ & 30 & 545 & 430 & 115 & 96,6 & 18 & 14 & 4 & 43,10 & 37,16 & 5,94 & $-9,50$ \\
\hline \multirow{4}{*}{\multicolumn{2}{|c|}{$\begin{array}{l}\text { Média } \\
\text { Desvio padrão } \\
\text { Mínimo } \\
\text { Máximo }\end{array}$}} & 31,27 & 549,80 & 480,13 & 69,67 & 77,55 & 17,13 & 12,67 & 4,47 & 42,80 & 39,20 & 3,60 & $-5,18$ \\
\hline & & 7,27 & 25,28 & 33,66 & 31,31 & 24,98 & 2,42 & 2,50 & 2,07 & 1,09 & 1,78 & 1,41 & 2,47 \\
\hline & & 24,00 & 507,00 & 430,00 & 20,00 & 41,10 & 14,00 & 9,00 & 1,00 & 41,61 & 36,80 & 1,63 & $-9,50$ \\
\hline & & 46,00 & 596,00 & 534,00 & 129,00 & 124,00 & 22,00 & 18,00 & 9,00 & 45,12 & 42,93 & 6,52 & $-2,25$ \\
\hline
\end{tabular}

$\mathrm{ECC}=$ espessura central corneana; $\Delta \mathrm{ECC}=\mathrm{ECC}$ pré $-\mathrm{ECC}$ pós; $\mathrm{Ablação=}$ profundidade de ablação estimada pelo laser; $\mathrm{PIO}=$ pressão intraocular; $\Delta \mathrm{PIO}=\mathrm{PIO}$ pré $-\mathrm{PIO}$ pós; $\mathrm{K}=$ ceratometria simulada média; $\Delta \mathrm{K}=\mathrm{K}$ pré $-\mathrm{K}$ pós; $\mathrm{EE}=$ equivalente esférico tratado por laser; $\mathrm{D}=$ dioptrias 
significante entre as medidas da PIO, de K e da ECC pré e pósLASIK. $(P=0,0001)$.

Considerando os dados pré e pós-operatórios, o coeficiente de correlação de Pearson demonstrou uma moderada correlação entre ECC e PIO ( $R=0,56$ e $p=0,001)$ (Gráfico 1) e uma fraca correlação entre $\mathrm{K}$ e PIO ( $R=0,39$ e $p=0,033)$ (Gráfico 2). Além de não apresentar significância estatística, a profundidade de ablação teve uma fraca correlação com a PIO ( $R=0,36$ e $p=0,162)$ e com a variação da $\mathrm{PIO}(\mathrm{R}=0,04$ e $\mathrm{p}=0,887)$, no pós-operatório. Foi observado que para cada 1D corrigida, há uma subestimação, em média, de 1,06 \pm $0,59 \mathrm{mmHg}(0,11$ a 1,89 mmHg) (Tabela 2).

Quando aplicamos a fórmula descrita anteriormente, $80 \%$ dos olhos apresentaram a tonometria pós-operatória entre $\pm 2,50 \mathrm{mmHg}$ da pré-operatória. A tonometria corrigida pela fórmula foi 15,67 $\pm 2,92 \mathrm{mmHg}(11,67$ a 22,17 mmHg), no entanto, quando comparada com a tonometria pré-operatória, estas são estatisticamente diferentes $(p=0,0266)$ (Tabela 2).

\section{DISCUSSÃO}

O exame oftalmológico de rotina deve incluir a medida da PIO, sendo essencial para triagem, tratamento precoce e manejo de várias doenças oculares. A forma mais comum de se aferir a PIO é com o tonômetro de aplanação de Goldmann (TAG), que se difun-

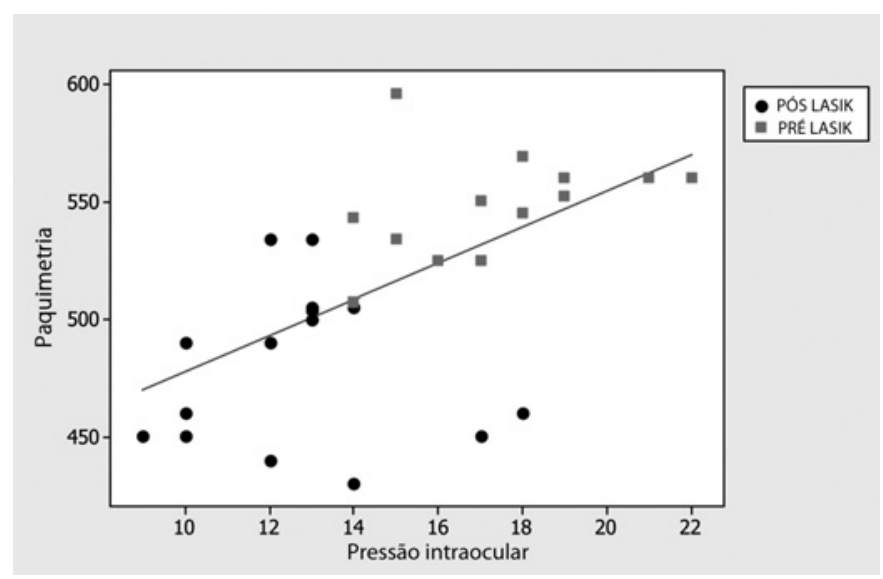

Gráfico 1.Correlação entre espessura central corneana e pressão intraocular, dados pré e pós-operatórios. $(R=0,56$ e $p=0,001)$.

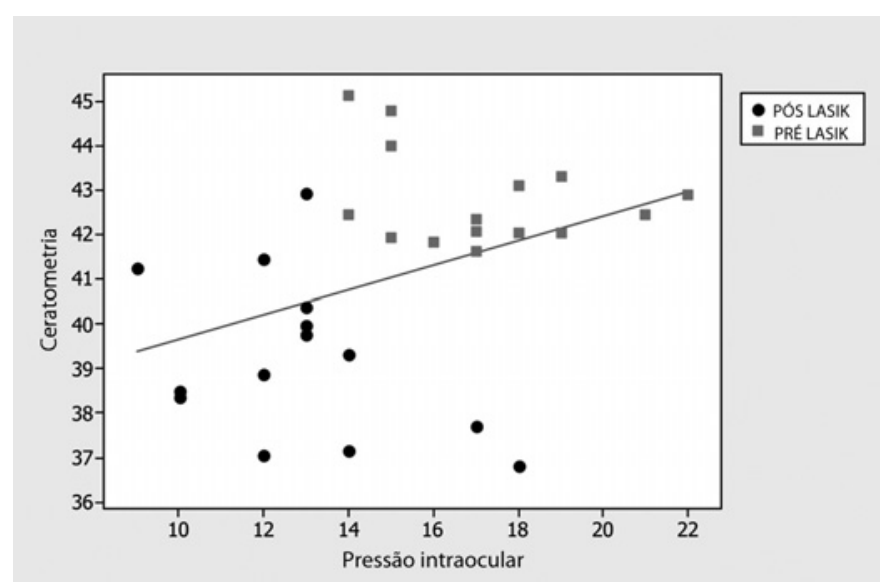

Gráfico 2. Associação da ceratometria simulada média e pressão intraocular, dados pré e pós-operatórios. $(R=0,39$ e $p=0,001)$. diu e teve rápida aceitação logo após sua introdução no mercado na década de 50, mantendo-se como padrão ouro até os dias de hoje. O próprio Goldmann reconheceu as limitações de seu invento, com pressuposição para ECC em torno de $500 \mu \mathrm{m}$, cujo desvio seria provocador de vieses de aferição(6).

Vários estudos demonstraram que a ECC exerce influência na tonometria de aplanação subestimando e superestimando o valor pressórico real em córneas finas e espessas, respectivamente ${ }^{(3-5)}$. A ECC em população saudável tem distribuição Gaussiana com média de $520 \mu \mathrm{m}^{(7)}$, embora, essa possa variar muito de acordo com a população estudada. A partir de equação de regressão linear, uma PIO real de $20 \mathrm{mmHg}$ produziria subestimação de $5,2 \mathrm{mmHg}$ em olhos com ECC de $470 \mu \mathrm{m}$ e superestimação de $4,7 \mathrm{mmHg}$ se a ECC fosse de $590 \mu \mathrm{m}$ com média de erro de $5 \mathrm{mmHg}$ para cada $70 \mu \mathrm{m}$ de variação na ECC ${ }^{(8)}$. Outro estudo encontrou uma média menor (3,5 mmHg) para cada $70 \mu \mathrm{m}$ de diminuição na ECC (5). Não está elucidado se córneas iatrogenicamente finas pós-LASIK têm a mesma influência na leitura da pressão intraocular que as córneas naturalmente finas ${ }^{(2)}$.

Procedimentos ceratorrefrativos (PRK e LASIK) diminuem a ECC, produzindo uma falsa medida da PIO. A redução na medida da PIO após cirurgia refrativa varia em torno de $2,5 \mathrm{mmHg}(0,5 \mathrm{mmHg} / 1 \mathrm{D}$ de miopia) $)^{(9-10)}$.

Já está claro e difundido que alterações na ECC e no K induzem a leituras errôneas ou falseadas por TAG. Entretanto, a diminuição na estabilidade corneana provocada pela confecção do flap e suas consequências na PIO ainda não está definida(3).

Com todos esses fatores explanados, torna-se imprescindível exame pré-operatório minucioso e técnica cirúrgica refrativa precisa, mesmo porque há muitos jovens sendo submetidos a esse procedimento com um futuro oftalmológico ainda incerto. Os dados préoperatórios se tornarão fundamentais na conduta e acompanhamento desses pacientes e na ausência desses exames, poder-se-á fazer uso de fórmulas para a estimativa da PIO real. Embora até hoje não tenha sido demonstrado uma validade precisa de nenhuma fórmula já desenvolvida.

No presente estudo, a média de diminuição da PIO pós-LASIK foi de 4,47 \pm 2,07 $\mathrm{mmHg}$, sendo uma diferença estatisticamente significante $(p=0,0001)$. Assim como as médias de $\mathrm{K}$ e de ECC pré e pós-LASIK ( $p=0,0001)$. Mostrando que a cirurgia realmente provoca alterações nessas variáveis, que podem influenciar a aferição da PIO pós-operatória.

Na análise univariada, não houve correlação entre a profundidade de ablação de a variação da $\mathrm{PIO}(\mathrm{R}=0,04$ e $\mathrm{p}=0,887)$, houve fraca correlação entre $\mathrm{K}$ e $\mathrm{PIO}(\mathrm{R}=0,39$ e $\mathrm{p}=0,033)$, e houve moderada correlação entre ECC a PIO ( $R=0,56$ e $p=0,001)$. O estudo original demonstrou moderada correlação da PIO com ECC e com K ${ }^{(3)}$.

A cada 1 D corrigida, houve subestimação de 1,06 $\pm 0,59 \mathrm{mmHg}$. Nota-se que, apesar de haver diminuição da PIO em todos os olhos, existe grande variação na influência de cada dioptria tratada na PIO (variação de 0,11 a 1,89 mmHg por dioptria tratada).

Utilizando a fórmula proposta, $80 \%$ dos olhos estudados apresentaram PIO entre $\pm 2,5 \mathrm{mmHg}$ da pré-operatória, no estudo original esta taxa foi de $70,3 \% \%^{(3)}$. No entanto, estas amostras continuam sendo diferentes $(p=0,0266)$. Nota-se que a aplicação desta fórmula levou a hipoestimação da PIO de 4,40 e 6,48 mmHg nos dois olhos de um mesmo paciente que apresentava 21 e $22 \mathrm{mmHg}$ no pré-operatório. Em outro olho, a aplicação da fórmula, levou a hiperestimação de 3,17 mmHg.

O pequeno tamanho da amostra, além da utilização de dois olhos de um mesmo paciente em 14 dos 15 olhos estudados e a medida da TAG apenas um vez nos períodos pré e pós-operatórios, podem ter influenciado nos resultados observados neste estudo.

\section{CONCLUSÕES}

Nessa amostra estudada, os olhos submetidos a LASIK apresentaram PIO pós-operatória menor do que a pré-operatória. A PIO pôde ser moderadamente correlacionada com a ECC e fracamente 
Tabela 2. Estimativa da pressão intraocular

\begin{tabular}{|c|c|c|c|c|c|c|c|c|}
\hline Olho & Sexo & Idade & $\begin{array}{l}\text { PIO Pré } \\
(\mathrm{mmHg})\end{array}$ & $\begin{array}{l}\text { PIO Pós } \\
\text { (mmHg) }\end{array}$ & $\begin{array}{l}\text { Diminuição por } \\
\text { Dioptria (mmHg) }\end{array}$ & $\begin{array}{c}\Delta \text { PIO Estimada } \\
(\mathrm{mmHg})\end{array}$ & $\begin{array}{l}\Delta \text { PIO Estimada vs } \\
\text { PIO Pré }(\mathrm{mmHg})\end{array}$ & $\begin{array}{c}\Delta \text { PIO Pós vs } \\
\text { PIO Pré }(\mathrm{mmHg})\end{array}$ \\
\hline OD & $\mathrm{F}$ & 28 & 15 & 10 & 1,43 & 13,69 & 1,31 & $-5,00$ \\
\hline OE & $\mathrm{F}$ & 28 & 16 & 10 & 1,71 & 13,60 & 2,40 & $-6,00$ \\
\hline OD & $\mathrm{F}$ & 27 & 14 & 9 & 0,83 & 11,67 & 2,33 & $-5,00$ \\
\hline OD & $M$ & 36 & 21 & 14 & 1,40 & 16,60 & 4,40 & $-7,00$ \\
\hline OE & $M$ & 36 & 22 & 13 & 1,89 & 15,52 & 6,48 & $-9,00$ \\
\hline OD & $\mathrm{F}$ & 33 & 17 & 12 & 1,11 & 14,98 & 2,02 & $-5,00$ \\
\hline OE & $\mathrm{F}$ & 33 & 14 & 10 & 0,89 & 13,13 & 0,87 & $-4,00$ \\
\hline OD & $\mathrm{F}$ & 24 & 17 & 13 & 1,78 & 15,36 & 1,64 & $-4,00$ \\
\hline OE & $\mathrm{F}$ & 24 & 17 & 13 & 1,78 & 15,23 & 1,77 & $-4,00$ \\
\hline OD & $M$ & 46 & 19 & 17 & 0,25 & 20,98 & $-1,98$ & $-2,00$ \\
\hline OE & $M$ & 46 & 18 & 12 & 0,75 & 16,37 & 1,63 & $-6,00$ \\
\hline OD & $\mathrm{F}$ & 24 & 15 & 12 & 1,00 & 13,40 & 1,60 & $-3,00$ \\
\hline OE & $\mathrm{F}$ & 24 & 15 & 13 & 0,57 & 13,86 & 1,14 & $-2,00$ \\
\hline OD & $M$ & 30 & 19 & 18 & 0,11 & 22,17 & $-3,17$ & $-1,00$ \\
\hline OE & M & 30 & 18 & 14 & 0,42 & 18,46 & $-0,46$ & $-4,00$ \\
\hline Média & & & 17,13 & 12,67 & 1,06 & 15,67 & 1,47 & $-4,47$ \\
\hline Desvio padrão & & & 2,42 & 2,50 & 0,59 & 2,92 & 2,29 & 2,07 \\
\hline Mínimo & & & 14,00 & 9,00 & 0,11 & 11,67 & $-3,17$ & $-9,00$ \\
\hline Máximo & & & 22,00 & 18,00 & 1,89 & 22,17 & 6,48 & $-1,00$ \\
\hline
\end{tabular}

$\mathrm{PIO}=$ pressão intraocular; Diminuição por Dioptria= diminuição da PIO por dioptria tratada em relação ao pré-operatório; PIO Estimada= PIO estimada pela aplicação da fórmula: [PIO real $=\mathrm{PIO}$ aferida $+(540-\mathrm{ECC}) / 71+(43-\mathrm{K}) / 2,7+0,75 \mathrm{mmHg}]^{(3)} . \Delta \mathrm{PIO}$ Estimada vs PIO Pré $=$ Diferença entre a PIO estimada e a PIO pré-operatória; $\Delta$ PIO Pós vs PIO Pré= diferença entre a PIO pós-operatória e a PIO pré-operatória. Dados com valores absolutos maiores que 2,50 mmHg estão em negrito

com a K. Não houve correlação entre a profundidade de ablação e a variação da PIO, no pós-operatório. Usando a fórmula proposta pudemos averiguar que $80 \%$ dos pacientes apresentaram PIO entre $\pm 2,50 \mathrm{mmHg}$ da pré-operatória.

\section{REFERÊNCIAS}

1. Pallikaris IG, Siganos DS. Excimer laser in situ keratomileusis and photorefractive keratectomy for correction of high myopia. J Refract Corneal Surg. 1994;10(5):498-510.

2. Ruangvaravate N, Thuangtong A, Kosrirukvongs P, Prabhasawat P, Booranapong W, Srivannaboon $\mathrm{S}$. Tonometry after laser in situ keratomileusis treatment: a preliminary study in Thai patients. J Med Thai. 2005:88(3):340-4.

3. Kholhaas M, Spoerl E, Boehm AG, Pollack K. A correction formula for the real intraocular pressure after LASIK for the correction of myopic astigmatism. J Refract Surg. 2006;22(3):263-7.
4. Grodum K, Heijl A, Bengtsson B. Refractive error and glaucoma. Acta Ophthalmol Scand. 2001;79(6):560-6. Comment in: Acta Ophthalmol Scand. 2002;80(2):230-1.

5. Whitacre MM, Stein RA, Hassanein K. The effect of corneal thickness on applanation tonometry. Am J Ophthalmol. 1993;115(5);592-6.

6. Brandt JD. Corneal thickness in glaucoma screening, diagnosis, and management. Curr Opin Ophthalmol. 2004;15(2):85-9.

7. Hansen FK. A clinical study of normal human central thickness. Acta Ophthalmol (Copenh) 1971;49(1):82-9.

8. Ehlers N, Hansen FK. Central corneal thickness in lower-tension glaucoma. Acta Ophthalmol (Copenh). 1974:52(5):740-6.

9. Emara B, Probst LE, Tingey DP, Kennedy DW, Willms LJ, Machat J. Correlation of intraocular pressure and central corneal thickness in normal myopic eyes and after laser in situ keratomileusis. J Cataract Refract Surg. 1998;24(10):1320-5.

10. Montes-Micó R, Charman WN. Intraocular pressure after laser myopic refractive surgery Ophthalmic Physiol Opt. 2001;21(3):228-35.

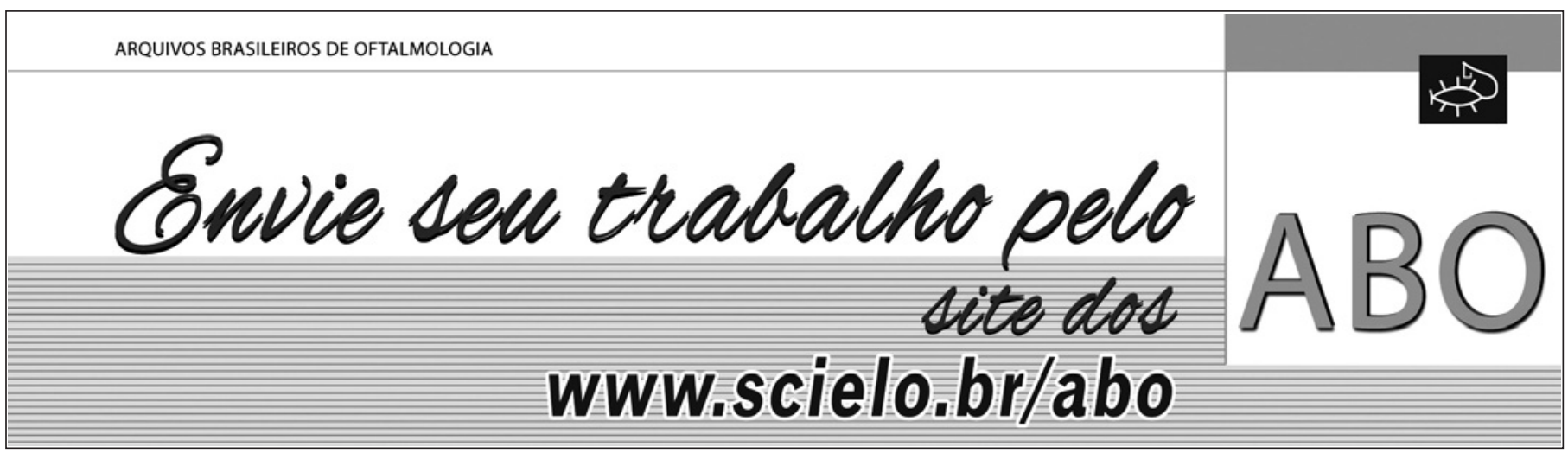

